\title{
Preferential depletion of a splenic marginal zone-like peripheral blood CD27+B220- memory B cell population in HIV-I infected individuals
}

\author{
M Morrow ${ }^{1}$, A Valentin11, R Little², R Yarchoan² and GN Pavlakis*1
}

Address: ${ }^{1}$ Human Retrovirus Section, Vaccine Branch, Center for Cancer Research, National Cancer Institute at Frederick, Frederick Maryland, USA and ${ }^{2} \mathrm{HIV}$ and AIDS Malignancy Branch, Center for Cancer Research, National Cancer Institute, Bethesda, Maryland, USA

* Corresponding author

from 2006 International Meeting of The Institute of Human Virology

Baltimore, USA. |7-2I November, 2006

Published: 21 December 2006

Retrovirology 2006, 3(SuppI I):S97 doi:10.1186/1742-4690-3-SI-S97

(c) 2006 Morrow et al; licensee BioMed Central Ltd.

In addition to CD4+ T cell depletion, HIV-1 infection is characterized by changes in humoral immunity including hypergammaglobulinemia, polyclonal B cell activation and loss of circulating memory B lymphocytes. Expression of the CD45 isoform B220 has recently been described on CD27- and a subset of CD27+ human B lymphocytes. The aim of this study was to evaluate B cell defects in HIV infection in more detail. We studied the frequency of memory B cells, based on the expression of B220, in HIV infected individuals $(\mathrm{n}=27)$ and healthy controls $(\mathrm{n}=$ 22) and characterized their functional properties. RNA expression of TLR9 and activation-induced cytidine deaminase (AICD) was analyzed by Real-time PCR from B cells sorted according to their memory phenotype. In addition, B-cell proliferative responses and immunoglobulin secretion in response to SAC and ODN treatment were monitored.

We found that the previously described reduction in the frequency of CD27+ B cells in HIV-infected individuals affects preferentially the subset characterized by the lack of B220 expression. The proportion of CD27+B220- B cells expressing surface IgD (28.8\%) and IgM (29.5\%) was reduced in the HIV+ group compared to healthy controls (53.9\% and 59.8\%, respectively). This depletion did not correlate with either CD4 counts or viral load, and was not reversed by antiretroviral therapy. We found that $\mathrm{CD} 27+\mathrm{B} 220$ - B cells have a splenic marginal zone like immunophenotype (IgMhiIgDloCD21+CD23-), express
TLR9, and proliferate and secrete IgG and IgM in response to B cell specific ODN. In contrast, CD27+B220+ B cells are IgMloIgDhiCD21+CD23+, express AICD and proliferate in response to SAC but do not secrete immunoglobulins.

The lack of B220 expression on CD27+ B cells defines a distinct memory B cell compartment that is preferentially depleted in HIV-infected individuals. The phenotype, pattern of gene expression and functional properties of the preferentially depleted $\mathrm{CD} 27+\mathrm{B} 220-\mathrm{B}$ cells suggest that these cells are splenic marginal zone-like circulating memory B cells. The lack of these cells in HIV patients may play an important role in the defective immunity against Tindependent pathogens and may impair the humoral control of HIV-1 propagation. 